\title{
Global MHD simulations of disk-magnetosphere interactions: accretion and outflows
}

\author{
M. M. Romanova ${ }^{1}$, R. V. E. Lovelace $^{2}$, G. V. Ustyugova $^{3}$ \\ and A. V. Koldoba ${ }^{3}$ \\ ${ }^{1}$ Department of Astronomy, Cornell University, \\ Ithaca, NY 14853-6801 \\ email: romanova@astro.cornell.edu \\ ${ }^{2}$ Department of Astronomy and Department of Applied and Engineering Physics, \\ Cornell University, Ithaca, NY 14853-6801 \\ email: RVL1@cornell.edu \\ ${ }^{3}$ Keldysh Institute of Applied Mathematics \\ Russian Academy of Sciences, Moscow, Russia \\ email: ustyugg@rambler.ru; koldoba@rambler.ru
}

\begin{abstract}
We outline recent progress in understanding the accretion of plasma to rotating magnetized stars obtained from global axisymmetric (2D) and 3D magnetohydrodynamic (MHD) simulations in three main areas: (1.) Formation of jets from disk accretion onto rotating magnetized stars: From simulations where the viscosity and magnetic diffusivity within the disk are described by alpha models, we find long-lasting conical outflows/jets from the disk/magnetosphere boundary in both the case where the star is slowly rotating and where it is rapidly rotating (the "propeller regime"). Most of the mass flux in the outflows is in a hollow cone but inside this cone there is a low-density high-velocity magnetically dominated flow along the open polar field lines of the star. The outflows occur under conditions where the poloidal magnetic flux of the star is bunched up by the accretion disk near the disk/magnetosphere boundary. Recent simulations show that the conical outflows become well-collimated for axial distances of $\lesssim 20$ times the inner disk radius. Exploratory 3D simulations show that conical winds are axisymmetric about the rotational axis (of the star and the disk), even when the dipole field of the star is significantly misaligned. (2.) Formation of intrinsically one-sided jets from disk accretion to rotating magnetized stars: There is strong observational evidence for an asymmetry between the approaching and receding jets from a number of young stars. We discuss the first MHD simulations of the formation asymmetric or one-sided jets arising from disk accretion to a rotating star with an asymmetric (dipole plus quadrupole) magnetic field. (3.) Global axisymmetric and 3D simulations of the magnetorotational instability (MRI) in disk accretion onto magnetized stars: In the axisymmetric simulations we observe cases where there is episodic or quasi-periodic burst of accretion similar to that observed in one $\mathrm{X}$ ray source. In 3D MHD simulations of accretion onto stars with tilted dipole fields using our Godunov-type code based on the "cubed sphere" grid we find that the density distribution is much less smooth than in the case of the laminar accretion flow described by $\alpha$-viscosity. Instead, large turbulent cells dominate the flows and are strongly elongated in the azimuthal direction.
\end{abstract}

Keywords. accretion, accretion disks; MHD; stars: magnetic fields 


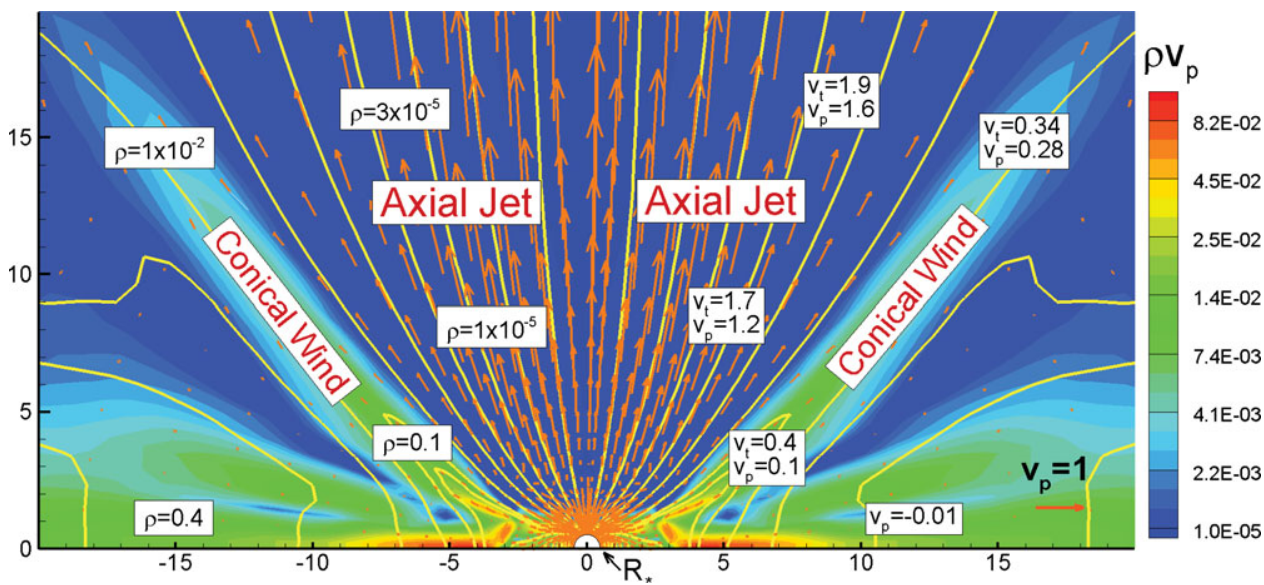

Figure 1. Matter flux $\rho v_{p}$ (background), sample field lines, and poloidal velocity vectors in a propeller-driven outflow at time $T=1400$ (rotation periods at the inner disk radius). Sample numerical values are given for the poloidal $v_{p}$ and total $v_{t}$ velocity, and for the density $\rho$ for different parts of the simulation region. Unit density corresponds to $\rho_{0}=4.1 \times 10^{-13} \mathrm{gcm}^{-3}$

\section{Outflows/jets from the disk-magnetosphere boundary}

Outflows and jets are observed from many disk accreting objects ranging from young stars to systems with white dwarfs, neutron stars, and black holes (e.g., Livio 1997). In addition to steady outflows there are episodic outbursts associated with periods of enhanced accretion (e.g., Cabrit et al. 1990). A large body of observations exists for outflows from young stars at different stages of their evolution, ranging from protostars, where powerful collimated outflows are observed, to classical T Tauri stars (CTTSs), where the outflows are weaker and often less collimated (see review by Ray et al. 2007). Different theoretical models have been proposed to explain the outflows from protostars and CTTSs (see review by Ferreira, Dougados, \& Cabrit 2006). The models include those where the outflow originates from a radially distributed disk wind (Königl \& Pudritz 2000; Casse \& Keppens 2004) or from the innermost region of the accretion disk (Lovelace, Berk \& Contopoulos 1991). Further, there is the X-wind model (Shu et al. 1994) where most of the outflow originates from the disk-magnetosphere boundary. The maximum velocities in the outflows are usually of the order of the Keplerian velocity of the inner region of the disk. This favors the models where the outflows originate from the inner disk region, or from the disk-magnetosphere boundary (if the star has a dynamically important magnetic field).

We have carried out systematic axisymmetric and limited 3D MHD simulations of outflows/jets from the disk-magnetosphere boundaries of rotating magnetized stars (Romanova et al. 2009). The disk is at a low temperature and is modeled by an alpha viscosity and a second alpha magnetic diffusivity and a high temperature low-density disk corona. We found outflows in two main cases: (1) where the star rotates slowly but the poloidal field lines of the star are bunched at the disk-magnetosphere boundary, and (2) where the star rotates rapidly in the propeller regime and the condition for bunching is also satisfied. In both cases, two-component outflows are observed as shown in Figure 1. One component originates near the disk/magnetosphere boundary and has a narrow-shell conical shape, and we term it a "conical wind". The other component is a magnetically dominated high-velocity low-density wind which flows along the open polar field lines of the star and it is referred to as the "jet." The energy and angular momentum fluxes in the two components are typically comparable. Exploratory 3D simulations show that 
conical winds are axisymmetric about the rotational axis (of the star and the disk), even when the dipole field of the star is significantly misaligned.

Figure 2 shows the much stronger magnetic collimation found in our recent simulations for the case of a lower density corona and a significantly larger simulation region (Lii et al. 2010).

\section{One-sided outflows/jets from rotating stars with complex magnetic fields}

There is clear evidence, mainly from Hubble Space Telescope (HST) observations, of the asymmetry between the approaching and receding jets from a number of young stars. The objects include the jets in HH 30 (Bacciotti et al. 1999), RW Aur (Woitas et al. 2002), TH 28 (Coffey et al. 2004), and $\mathrm{LkH} \alpha 233$ (Pererin \& Graham 2007). Specifically, the radial speed of the approaching jet may differ by a factor of two from that of the receding jet. For example, for RW Aur the radial redshifted speed is $\sim 100 \mathrm{~km} / \mathrm{s}$ whereas the blueshifted radial speed is $\sim 175 \mathrm{~km} / \mathrm{s}$. The mass and momentum fluxes are also significantly different for the approaching and receding jets in a number of cases. It is possible that the observed asymmetry of the jets could be due to say differences in the gas densities on the two sides of the source. However, there is substantial observational evidence that young stars often have complex magnetic fields consisting of dipole, quadrupole, and higher order poles possibly misaligned with respect to each other and the rotation axis (e.g., Donati et al. 2008).

We have carried out MHD simulations of the formation of conical winds for the axisymmetric dipole/quadrupole field combinations for cases where the outflows are not required to be symmetrical about the equatorial plane (Lovelace et al. 2010). The lefthand panel of Fig. 3 shows the vacuum field and the right-hand panel a sample result from our simulations.

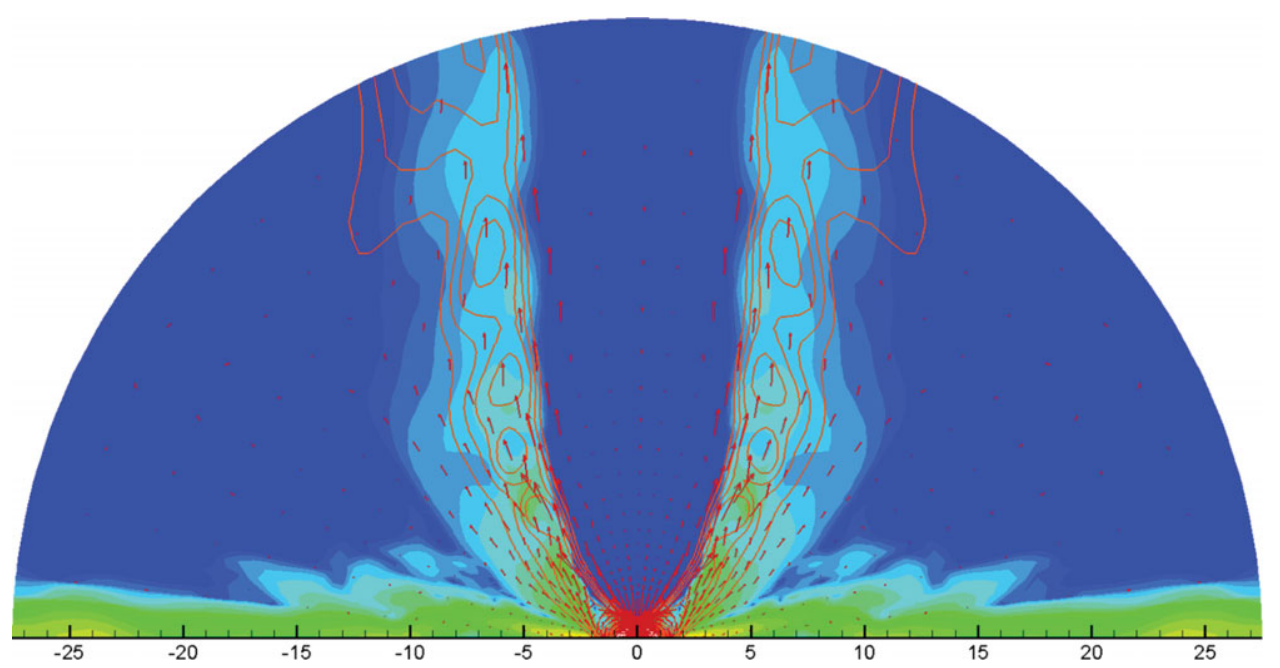

Figure 2. Axisymmetric simulations for a lower coronal density $\left(3 \times 10^{-4} \rho_{\text {disk }}\right)$ and a larger simulation region show that the toroidal magnetic field gives strong collimation of the outflow (Lii et al. 2011). 


\section{Global MHD simulations of the MRI driven accretion onto magnetized stars}

It is widely thought that the magnetorotational instability (MRI) in ionized weaklymagnetized accretion disks gives rise to turbulence which is responsible for the fast outward transport of angular momentum in the disk (Balbus \& Hawley 1991, 1998). The MRI driven accretion has been observed in different simulations, local shearing box models, global axisymmetric models, and global 3D simulations (e.g., Balbus \& Hawley 1998; Hawley et al. 2001; Beckwith, Hawley, \& Krolik 2009). Fortuitously, the MRI turbulence found in simulations can be described approximately by an Shakura \& Sunyaev (1973) $\alpha$ coefficient of $\sim 0.01-0.1$.

The global axisymmetric and 3D simulations of the MRI turbulence in disks has been done for the case of accretion disks around black holes and for cases where the initial magnetic field is inside the disk. However, the situation is different for the case of an accretion disk around a rotating star with a dynamically important magnetic field, or the case where the star has an effectively solid surface at which magnetic flux can accumulate and interact with an incoming MRI-driven flow. The details of such interactions are unknown and no simulations of such interaction have been done yet.

Long wavelength components ( $\gg$ disk half-thickness) of the MRI instability may be directly contributing to the observed time variations in some sources. A number of anomalous millisecond pulsars (AMP) show episodic or quasi-periodic flares in the tails of their bursts. For example, the pulsar SAX J1808.4-3658 shows flaring activity with a quasiperiod of about $1 \mathrm{~Hz}$ (Patruno et al. 2009).

Recently, we have found interesting variability in axisymmetric simulations of the MRIdriven accretion onto magnetized star (Romanova et al. 2011a). The simulations show that the disk-magnetosphere interaction depends on the orientation of the poloidal magnetic field inside the disk relative to the direction of the $B$-field inside the magnetosphere. This field is much smaller than the azimuthal field, but it determines the character of
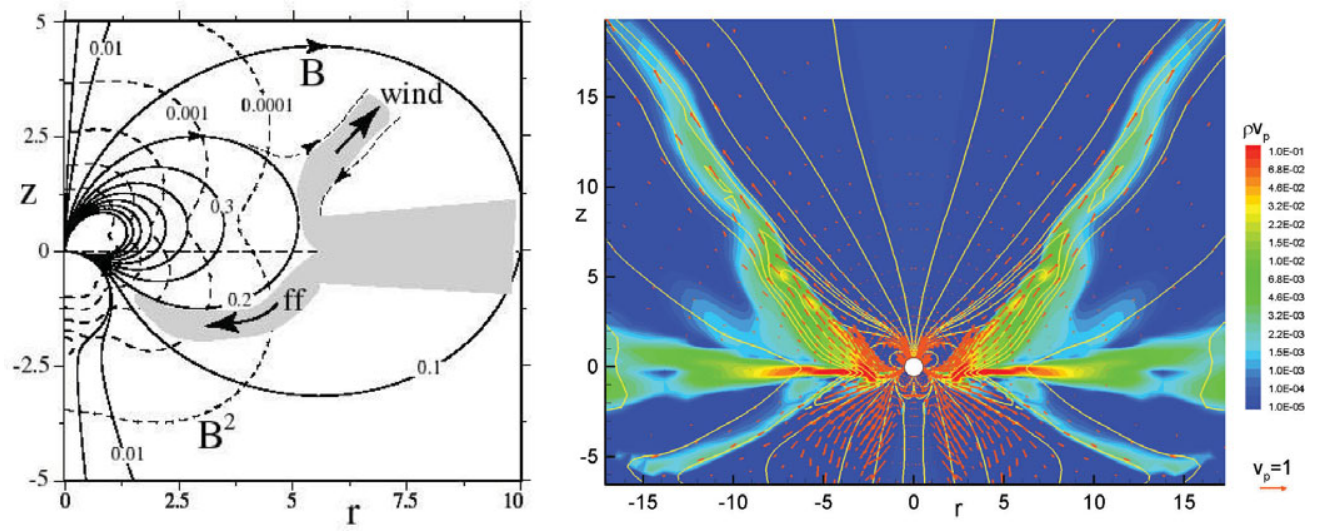

Figure 3. The left-hand panel shows the magnetic field lines $\Psi(r, z)=$ const and constant magnetic pressure lines for the case of an aligned dipole and quadrupole field where the flux function is $\Psi=\mu_{d} r^{2} / R^{3}+(3 / 4) \mu_{q} z r^{2} / R^{5}$, where $R^{2}=r^{2}+z^{2}$ and $\mu_{d}$ is the dipole moment and $\mu_{q}$ is the quadrupole moment. Roughly, $\mu_{q} / \mu_{d}$ is the distance at which dipole and quadrupole fields are equal. The funnel flow (ff) and the wind in this figure are suggested. The dashed lines are constant values of $\mathbf{B}^{2}$. The right-hand panel gives snapshot of the outflow in the case where $\tilde{\mu}_{d}=10, \tilde{\mu}_{q}=20$ at $t=50$ as discussed by Lovelace et al. (2010). The color background shows the matter flux-density and the lines are the poloidal field lines. The vectors show the poloidal velocity. 

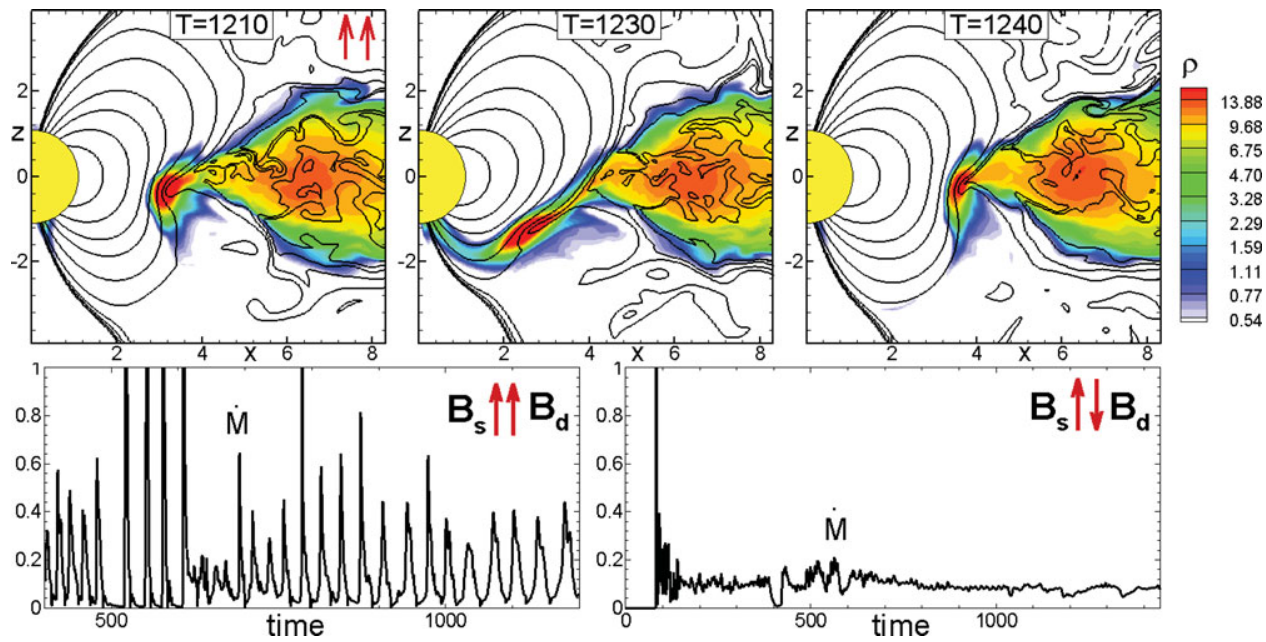

Figure 4. The top panels show three moments of time for a flaring episode of accretion for a case where the seed magnetic field of the disk is parallel to that of the star at the disk-magnetosphere boundary. The bottom panels show the accretion rate onto the star for cases of parallel (left panel) and antiparallel (right panel) fields. From Romanova et al. (2011a).

the disk-magnetosphere interaction and variability of the accretion rate onto the star. Namely, in the case where the poloidal field in the disk is parallel to that of the star, the matter accumulates for a significant time, then it goes to a funnel flow and accretes to the star. Fig. 4 shows episodes of plasma accumulation (top left panel), accretion (top middle panel) and new accumulation (top right panel). The corresponding accretion rate onto the star is shown in the bottom panel of Fig. 4. The bottom right panel of Fig. 4 shows the opposite case where the poloidal field in the disk is anti-parallel to that of the star. The smooth light-curve reflects the fact that the field of the star and the disk have frequent events of reconnection where matter of the disk moves onto the field lines of the star more easily and the accretion is quasi-steady.

In further recent work we performed 3D MHD simulations of accretion onto stars with tilted dipole fields (Romanova et al. 2011b) using our Godunov-type code based on the "cubed sphere" grid (Koldoba et al. 2002). We observed in these simulations that the density distribution is much less smooth than in the case of the laminar accretion flow described by $\alpha$-viscosity. Instead, large turbulent cells dominate the flows and are
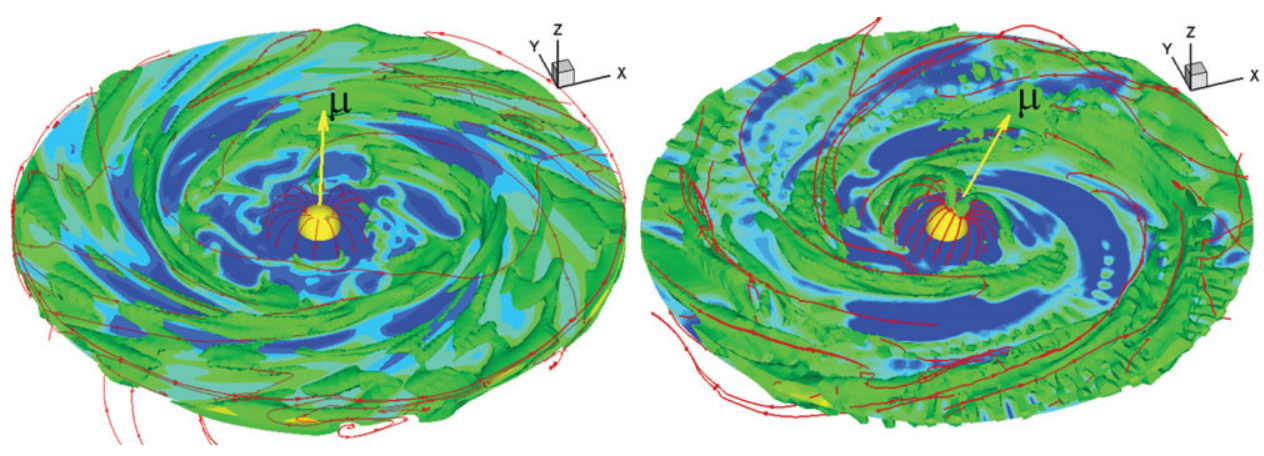

Figure 5. 3D MHD simulations of MRI-driven accretion onto a star with a dynamically-important magnetic field. Left panel: the dipole tilt angle is small, $\Theta=5^{\circ}$. Right panel: $\Theta=30^{\circ}$. From Romanova et al. (2011b). 
strongly elongated in the azimuthal direction. Fig. 5 shows the full 3D view of the MRIdriven disks in cases of very low tilt of the dipole (left panel) and high tilt (right panel). One can see that in the case of a tilted dipole, matter accretes in funnel streams but the streams are less ordered compared with cases of laminar $\alpha$-viscosity flow. Also, spiral paths of the flow are observed and we often see signs of one or two dominant spiral waves in the disk, and warping of the disk. Simulations were performed for different grid resolutions of our cubed sphere with the angular resolution varying from $61^{2}$ up to $91^{2}$ in each of 6 blocks. In addition, the grid was compressed in the vertical direction which made the initial vertical resolution of the disk of 110 cells across the disk. We observed that even at high resolution, large-scale structures are dominant.

We thank Dr. Alfio Bonanno for his lead in organizing this excellent symposium. This work was supported in part by NASA grants NNX08AH25G and NNX10AF63G and by NSF grant AST-0807129. MMR thanks NASA for use of the NASA High Performance Computing Facilities. AVK and GVU were supported in part by grant RFBR 09-0200502a, Program 4 of RAS.

\section{References}

Bacciotti, F., Eisloffel, J., \& Ray, T. P. 1999, A\&AA, 350, 917

Balbus, S. A. \& Hawley, J. F. 1991, ApJ, 376, 214

Balbus, S. A. \& Hawley, J. F. 1998, Rev. Mod. Phs., Volume 70, 1-53

Beckwith, K., Hawley, J. F., \& Krolik, J. H. 2009, ApJ, 707, 428

Cabrit, S., Edwards, S., Strom, S. E., \& Strom, K. M. 1990, ApJ, 354, 687

Coffey, D., Bacciotti, F., Woitas, J., Ray, T. P., \& Eislöffel, J. 2004, ApJ, 604, 758

Donati, J.-F. et al. 2008, MNRAS, 386, 1234

Ferreira, J, Dougados, C., \& Cabrit, S. 2006, A\& $A, 453,785$

Hawley, J. F, Balbus, S. A., \& Stone, J. M. 2001, ApJ Letters, 554, L49-L52

Koldoba, A. V., Romanova, M. M., Ustyugova, G. V., \& Lovelace, R. V. E. 2002, ApJ, 576, L53-L56

Königl, A. \& Pudritz, R. E. 2000, Protostars and Planets IV, Mannings, V., Boss, A. P., Russell, S. S. (eds.), University of Arizona Press, Tucson, p. 759

Lii, P., Romanova, M. M., \& Lovelace, R. V. E. 2010, in preparation

Livio, M. 1997, Accretion Phenomena and Related Outflows; IAU Colloquium 163. ASP Conference Series; Vol. 121; ed. D. T. Wickramasinghe; G. V. Bicknell; and L. Ferrario, p. 845

Lovelace, R. V. E., Romanova, M. M., Ustyugova, G. V., \& Koldoba, A. V. 2010, MNRAS, 408, 2083

Lovelace, R. V. E., Berk, H. L., \& Contopoulos, J. 1991, ApJ, 379, 696

Patruno, A., Wijnands, R., van der Klis, M. 2009b, ApJ, 698, L60-L63

Perrin, M. D.,\& Graham, J. R. 2007, ApJ, 670, 499

Ray, T., Dougados, C., Bacciotti, F., Eislffel, J., \& Chrysostomou, A. 2007, Protostars and Planets V, B. Reipurth, D. Jewitt, and K. Keil (eds.), University of Arizona Press, Tucson, p. 231

Romanova, M. M., Ustyugova, G. V., Koldoba, A. V., \& Lovelace, R. V. E. 2009, ApJ, 399, 1802

Romanova, M. M. et al. 2011a, in preparation

Romanova, M. M. et al. 2011b, in preparation

Shakura, N. I. \& Sunyaev, R. A. 1973, A\&\&A, 24, 337

Shu, F., Najita, J., Ostriker, E., Wilkin, F., Ruden, S., \& Lizano, S. 1994, ApJ, 429, 781

Woitas, J., Ray, T. P., Bacciotti, F., Davis, C. J., \& Eislöffel, J. 2002, ApJ, 580, 336 